\title{
Fine Facet Digital Watermark (FFDW) Mining From The Color Image Using Neural Networks
}

\author{
N.Chenthalir Indra \\ Assistant Professor, Computer science Department, \\ S.T.Hindu College, Nagercoi, Tamilnadu, India. 629001.
}

\author{
Dr. E . Ramaraj \\ Technology Advisor, M.K. University \\ Madurai, Tamilnadu,India.
}

\begin{abstract}
On hand watermark methods employ selective Neural Network techniques for watermark embedding efficiently. Similarity Based Superior Self Organizing Maps (SBS_SOM) a neural network algorithm for watermark generation. Host image is learned by the SBS_SOM neurons and the very fine RGB feature values are mined as digital watermark. Discrete Wavelet Transform (DWT) is used for watermark entrench. Similarity Ratio and PSNR values prove the temperament of the Fine Facet Digital Watermark (FFDW). The Proposed system affords inclusive digital watermarking system.
\end{abstract}

Keywords- Similarity based Superior SOM; Discrete Wavelet Transform; Digital watermark; embedding; PSNR.

\section{INTRODUCTION}

Digital image watermarking is a technique which embeds additional information called digital signature or watermark into the digital content in order to secure it [10]. A watermarking system is usually divided into four distinct steps, collecting digital watermark, embedding, attack and detection. Digital watermark may be any text, image, signals or any derived values. The proposed system generates watermark from host image by using Similarity Based Superior Self Organizing Maps (SBS_SOM) a neural network algorithm. In embedding, an algorithm accepts the host and the data to be embedded and produces a watermarked signal. The watermarked signal is then transmitted or stored.

If anybody makes a modification, this is called an attack. While the modification may not be malicious, the term attack arises from copyright protection application, where pirates attempt to remove the digital watermark through modification. There are many possible modifications, for example, lossy compression of the data, cropping an image or intentionally adding noise. This analysis verifies the robustness of watermark by smooth JPEG compression and adding standard noise. Detection (often called extraction) is an algorithm which is applied to the attacked signal to attempt to extract the watermark from it. In robust watermarking applications, the extraction algorithm should be able to produce the watermark again, even if the modifications were strong. In fragile watermarking, the extraction algorithm should fail if any change is made to the signal. Reference [8] uses BPN (Back Propagation Network) model to learn the relationship between the watermark and the watermarked image. Reference [5] used a full counter-propagation neural network (FCNN) for copyright protection where the ownership information was embedded and detected by a specific FCNN. Reference [1] proposed a new blind watermarking scheme in which a watermark was embedded into the DWT (Discrete Wavelet Transform) domain. It also utilized RBF Neural network to learn the characteristic of the image, using which the watermark would be embedded and extracted. Reference [4] presented a specific designed full counter-propagation neural network for digital image watermarking. Most of the systems used CPN, BPN and RBF algorithms. Various neural network algorithms were used to strengthen and retrieve the watermark values, but not for watermark value generation. The proposed system SBS_SOM a neural network algorithm was trained to generate digital watermark values from the image. The proposed system submits robust watermarking scheme. The section two gives detail about basic techniques Similarity based superior SOM which is used for watermark generation and detection and Discrete Wavelet Technique (DWT) for embedding. Section three describes the proposed system process. The experimental results are discussed in the section four. Section five summarizes the result.

\section{PROPOSED SYSTEM ENVIRONMENT}

Instead of using standard media as digital watermarks or mathematically derived watermarks the projected system imply a neural network algorithm called Self Organizing Maps (SOM). The SOM is a particular type of neural network used in clustering, visualization and abstraction [6]. It is an unsupervised competitive learner. Its learning pattern and order are unpredictable.

Existing applications on SOM express the benefits of using the SOM in applications with massive sets of data in finance, macroeconomics, medicine, biology, and other fields. The SelfOrganizing Maps have been used at the Research Center in such applications as: Automatic speech recognition, Clinical voice analysis, Monitoring of the condition of industrial plants, processes Cloud classification from satellite images, analysis of electrical signals from the brain organization and retrieval of data from large document collections. The outputs of SOM applications are highly visual, which assists the analyst in understanding the data's internal relationships.

However in the field of watermarking SOM was used for the process of embedding. The paper recommends the improvised SOM known as Similarity Based Superior SOM (SBS_SOM) for watermark generation. Conventional SOM and most of the extended SOM are using Euclidean-based distance metrics. The proposed SBS_SOM is more efficient 
than the conventional SOM. It uses Jaccard or Dice measures for winner node selection. So the training starts from maximum distance nodes, whereas SOM stars it's training from minimum distance nodes. Its factors such as weight initialization, learning rate, training epochs and neighborhood function are influencing the training nature of the network. These settings are standardized as per the rules. The same environment is created with conventional SOM for the analysis intention.

\section{A. Similarity Based Superior SOM}

References [2] and [3] reliably recommend Similarity based Self Organizing Maps neural network for image training. The Similarity based Superior Self Organizing Maps is the focal process to generate watermark. Significant initial factors are set by authorized person. Without knowing those values detection of watermark is impossible.

\section{1) Algorithm:}

Step0: Initialize weights with random method or by having previous knowledge of Pattern distribution. Set Topological neighborhood parameters. Set learning rate parameter

Step1: While stopping condition is false, do steps $2-8$

Step2: For each input vector $\mathbf{x}$, do steps $3-5$

Step3: For each $\mathbf{j}$, compute $d(j)$ by using any one of the following distance measures as in (1) or (2).

a) Jaccard Coefficient:

$$
\operatorname{sim}\left(x_{i}, w_{j}\right)=\frac{\sum_{h=1}^{k} x_{i h} w_{j h}}{\sum_{h=1}^{k} x_{i h}^{2}+\sum_{h=1}^{k} w_{j h}^{2}-\sum_{h=1}^{k} x_{i h} w_{j h}}
$$

b) Dice Coefficient:

$$
\operatorname{sim}\left(x_{i}, w_{j}\right)=\frac{2 \sum_{h=1}^{k} x_{i h} w_{j h}}{\sum_{h=1}^{k} x_{i h}^{2}+\sum_{h=1}^{k} w_{j h}^{2}}
$$

Step4: Find index $\mathbf{J}$ such that $\mathbf{d}$ ( $\mathbf{j})$ is a minimum.

Step5: For units $\mathbf{j}$ within a specified neighborhood of $\mathbf{J}$ and for all $\mathbf{I} \mathrm{W}_{\mathrm{ij}}($ new $)=\mathrm{W}_{\mathrm{ij}}($ old $)+\alpha\left[\mathrm{X}_{\mathrm{i}}-\mathrm{W}_{\mathrm{ij}}(\right.$ old $\left.)\right]$

Step6: Update learning rate

Step7: Reduce radius of topological at specified times

Step8: Test stopping condition.

The Learning Rate is a slowly decreasing function of time. The radius of the neighborhood around a cluster unit also decreases as the clustering process progresses. The updated weight network is well equipped with the host image neural structure. SBS_SOM exactly imitates human neural learning logic. Hence trivial imbalanced values can be identified through the analysis. These insignificant map elements in SBS_SOM network is determined and used as watermark values.

\section{B. Discrete Wavelet Transformation $(D W T)$}

The first Discrete Wavelet Transform (DWT) was invented by the Hungarian mathematician Alfred Haar. For an input represented by a list of $2^{n}$ numbers, the Haar wavelet transform may be considered to simply pair up input values, storing the difference and passing the sum. This process is repeated recursively, pairing up the sums to provide the next scale: finally resulting in $2^{n}-1$ differences and one final sum.

DWT decomposes input image into four components namely LL, HL, LH and HH. The lowest resolution level LL consists of the approximation part of the original image. The remaining three resolution levels consist of the detail parts and give the vertical high (LH), horizontal high (HL) and high $(\mathrm{HH})$ frequencies. In the proposed technique, embedding and extraction of watermark takes place in the high frequency component. For a one level decomposition, the discrete twodimensional wavelet transform of the image function $f(x, y)$ is found in [7] and [9]. DWT transform based watermarking scheme is robust against many common image attacks. The analysis results proved robustness very well.

\section{FinE FACET Digital WATERMARK SYSTEM (FFDW)}

In the proposed system host image is learned by the SBS_SOM neurons and the very fine RGB feature values are mined as digital watermark. Discrete Wavelet Transform (DWT) is used for watermark entrench.

\section{A. FFDW Generator}

1) Preprocessing:

1) Collect the input images. Select the image for watermarking.

2) Extract its RGB colors attributes in separate 2-D spaces.

\section{2) Apply SBS-SOM:}

1) Set three SBS_SOM network for representing Red, Green and Blue with three layers. (Input, hidden and output with two dimensional space matrix).

2) Initialize its weight vectors, neighborhood function, fix the epochs, set initial learning rate and fix reduction of learning rate for each epoch.

3) Train the RGB networks. Find the trained feature maps from output layer.

4) Find the difference between input values and trained values for each color. The resultant values are accepted as watermark values. Thus the first pass obtains three sets of watermarks.

\section{3) Embedding:}

1) Activate one-level DWT to original image's red vectors.

2) The red attribute watermark is embedded in to the high frequency component $\mathrm{HH}$ of DWT.

3) Execute inverse wavelet transform to obtain the watermarked red features.

4) Repeat the above three steps for other two green and blue colors too. 
5) By combining RGB watermarked plane the watermarked image will be obtained.

\section{B. FFDW Detector}

Projected watermarking proposal is capable of mine watermark information in the absence of the original image or secrete key. Hence it is unsighted watermarking.

1) Trigger one level DWT to the destination image and take away the embedded watermark from the HH sub band.

2) Regenerate watermark from the transferred image by using SBS_SOM neural logic as mentioned in the generation algorithm.

\section{PROFICIENCY ANALYSIS ON FFDW}

In order to prove the efficiency of SBS_SOM mined FFDW, the quality assessment experiments were done. It is proved that the proposed watermark technique has robustness, imperceptibility and authenticity.

For the experimentation, the digital watermark values with $64 \times 64$ size was generated from the host image which is be watermarked. Since the FFDW is mined through SBS_SOM neural network for each individual host image, it is unique. No one can predict the watermark values by means of common calculations. Minute details having inaptness were identified and collected as digital watermark. The RGB colors are trained individually. Hence the result organized three sets of digital watermarks.

Each 2-D plans are embedded by means of corresponding color FFDW values by using DWT. Subsequent to the embedding process the three 2-D planes are combined to form 3-D watermarked image. The Peak Signal to Noise Ratio (PSNR) as in (3), and Similarity Ratio (SR) as in (1), are estimated between host image and watermarked image. Besides, the same process was done with conventional SOM to compare the efficiency of the proposed SBS_SOM for watermarking. The experiment was carried out with the sample image gallery. The ten image samples and its corresponding watermarked image PSNR and SR values are tabularized in the Table 1.

$$
P S N R=10 \log _{10}\left(\frac{255^{2}}{M S E}\right)
$$

TABLE 1. PSNR \& SR OF 10 SAMPLE HOST IMAGES

\begin{tabular}{|l|c|c|c|c|}
\hline \multirow{2}{*}{ Host images } & \multicolumn{2}{|c|}{ SBS_SOM } & \multicolumn{2}{c|}{$\begin{array}{c}\text { Conventional } \\
\text { SOM }\end{array}$} \\
\cline { 2 - 5 } & $\boldsymbol{P S N R}(\boldsymbol{d B})$ & $\boldsymbol{S R}$ & $\boldsymbol{P S N R}(\boldsymbol{d B})$ & $\boldsymbol{S R}$ \\
\hline Cap girl & 53.7 & 1 & 32.4 & 0.88 \\
\hline Blue boy & 52.5 & 1 & 32.8 & 0.84 \\
\hline Chubby babby & 52.3 & 1 & 32.5 & 0.84 \\
\hline Cute baby & 50.3 & 1 & 30.5 & 0.83 \\
\hline Mary & 52.1 & 1 & 33.1 & 0.84 \\
\hline Green boy & 52.5 & 1 & 32.2 & 0.83 \\
\hline softy & 53.9 & 1 & 31.2 & 0.82 \\
\hline
\end{tabular}

\begin{tabular}{|l|l|l|l|l|}
\hline Been & 52.6 & 1 & 32.1 & 0.85 \\
\hline Infant & 51.7 & 1 & 33.2 & 0.85 \\
\hline Smiley & 55.6 & 1 & 30.5 & 0.81 \\
\hline
\end{tabular}
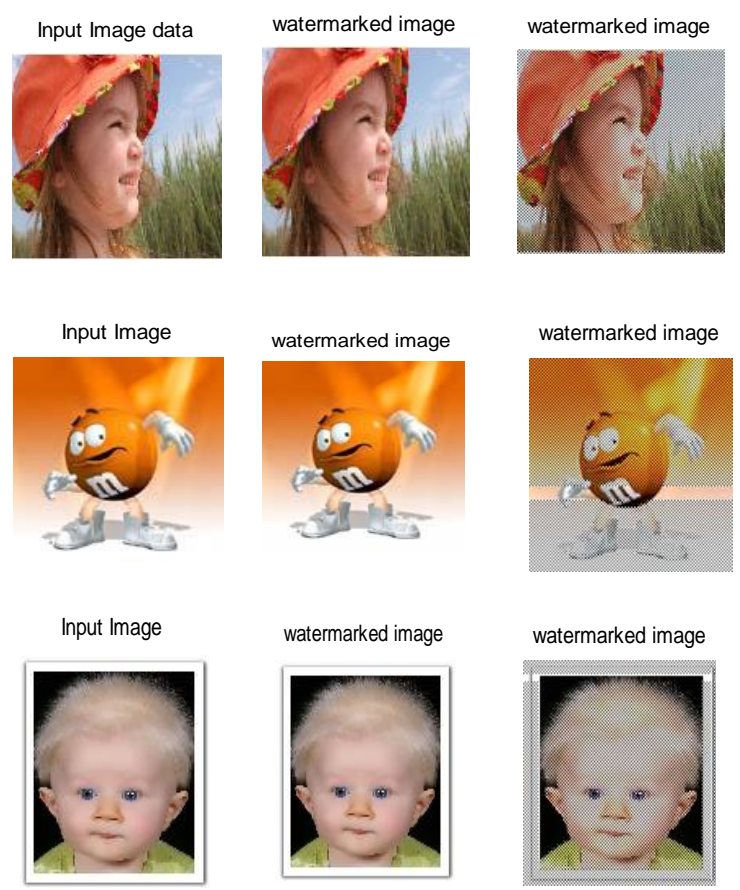

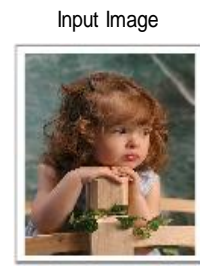

(a)

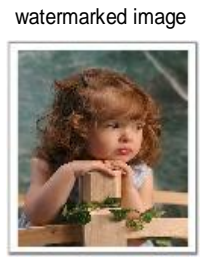

(b)

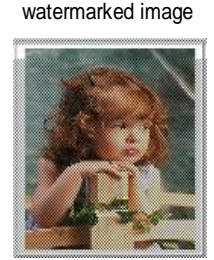

(c)
Figure 1. (a) Input images (b) SBS_SOM watermarked images (c) SOM watermarked images

Among those watermarking process four different color host images and its related watermarked images are given in the Fig. 1 (b) with SBS_SOM high level imperceptibility is proved. Visibly there is no degradation of image was found in the watermarked image. From the Table 1, it was proved that host image and watermarked image has fine similarity ratio ' 1 '. Well decent PSNR also found in proposed watermarking technique. Compressed and transferred watermarked image without any attack at the destination system receives imperceptible watermarked images. On the other hand SOM watermarking shows visible changes in the watermarked images. Fig. 1(c) column is the evident for SOM watermarking.

The robustness of the watermarking is checked by applying special kinds of noise like Gaussian, Poisson, Salt \& Pepper and Speckle. The attacked image of cap girl is shown in the Fig. 2. The watermarks are detected and extracted from the transferred watermarked image. The extracted watermark is 
compared with the original watermark by deducing PSNR value. The cap girl image watermark PSNR values with various attacks are mentioned in the Table 2.

TABLE 2. ROBUSTNESS OF WATERMARK

\begin{tabular}{|l|c|c|}
\hline \multirow{2}{*}{ Types of Attacks } & SBS-SOM & SOM \\
\cline { 2 - 3 } & $\boldsymbol{P S N R}(\boldsymbol{d} \boldsymbol{B})$ & $\boldsymbol{P S N R}(\boldsymbol{d} \boldsymbol{B})$ \\
\hline No Attack & 51.9 & 33 \\
\hline Compressed & 51.9 & 32 \\
\hline Gaussian & 51.2 & 32.1 \\
\hline Poisson & 51.6 & 33.2 \\
\hline Salt \& Pepper & 51.4 & 32.4 \\
\hline Speckle & 51.3 & 31.7 \\
\hline Damaged & 51.8 & 30.4 \\
\hline
\end{tabular}

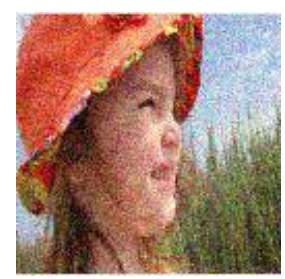

(a)

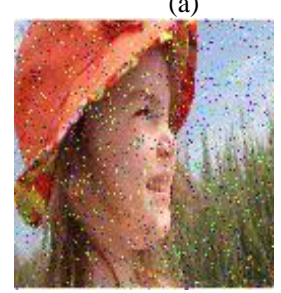

(c)

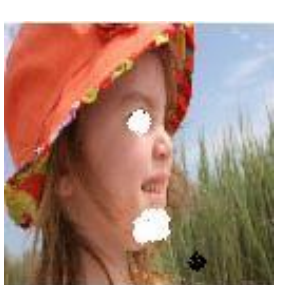

(e)

Figure 2. Noisy watermarked images (a) Gaussian noise (b) Poissian noise (c) Salt \& Pepper noise (d) Speckle noise (e) Damaged

One and the same results were evaluated in the case of other images too. The reasonable PSNR value even after noise attacks in the case of SBS_SOM proves the robustness of the watermarking. At the receiver end the attacked image can be strongly identified. For this the watermark is detected by authorized method and removed from the received image to get the original image back. Watermark removed image and host image PSNR and SR are calculated and tabulated in the Table 3 . The Table 3 clearly states that received watermarked image without any attack will produce exact host image values. The PSNR is at its maximum infinitive. This means that there is no difference between the values of host and watermark removed image at the receiver side.
At the receiver end the attacked image can be strongly identified. For this the watermark is detected by authorized method and removed from the received image to get the original image back. Watermark removed image and host image PSNR and SR are calculated and tabulated in the Table 3. It clearly states that received watermarked image without any attack will produce exact host image values. The PSNR is at its maximum infinitive. This means that there is no difference between the values of host and watermark removed image at the receiver side.

TABLE 3. QUALITY OF AN IMAGE AFTER THE REMOVAL OF WATERMARK

\begin{tabular}{|c|c|c|c|c|}
\hline \multirow[b]{2}{*}{ Attacks } & \multicolumn{2}{|c|}{ SBS_SOM } & \multicolumn{2}{|c|}{ SOM } \\
\hline & $\begin{array}{c}\text { PSNR } \\
(d B)\end{array}$ & $S R$ & $\begin{array}{c}\text { PSNR } \\
(d B)\end{array}$ & $S R$ \\
\hline No attack & INF & 1 & 35.2 & 0.87 \\
\hline Compressed & 46.7 & 0.999 & 34.6 & 0.81 \\
\hline Gaussian & 38.9 & 0.979 & 34.2 & 0.80 \\
\hline Poisson & 41.6 & 0.983 & 34.4 & 0.78 \\
\hline Salt \& Pepper & 39.1 & 0.970 & 33.8 & 0.82 \\
\hline Speckle & 38 & 0.972 & 34.3 & 0.79 \\
\hline Damaged & 42 & 0.981 & 33.5 & 0.82 \\
\hline
\end{tabular}

FFDW system does not affect the originality of the host image. And also the watermark is not detectable because the FFDW system uses unsupervised SBS_SOM for watermark generation. This neural network is unpredictable and the almost similar results can be evaluated only through the known parameter settings. Each and every parameter settings have their own authority on neural node organization. Hence using common statistical analysis or correlative attacks will never be able to find the watermark. Without proper watermark detection the original image cannot be restructured. Since FFDW system assures for secured watermarking.

The attacked images had lost their originality. Even though the watermark is robust the original image was not reconstructed. The Table 3 flaunts the trim down of PSNR and SR. For the copy right claim and authentication applications, the infinitive PSNR values are needed. FFDW system can be strongly proposed for authentic applications. The Fig. 3 visibly presents the secured image transformation with its selfgenerated digital watermarking quality assurance.

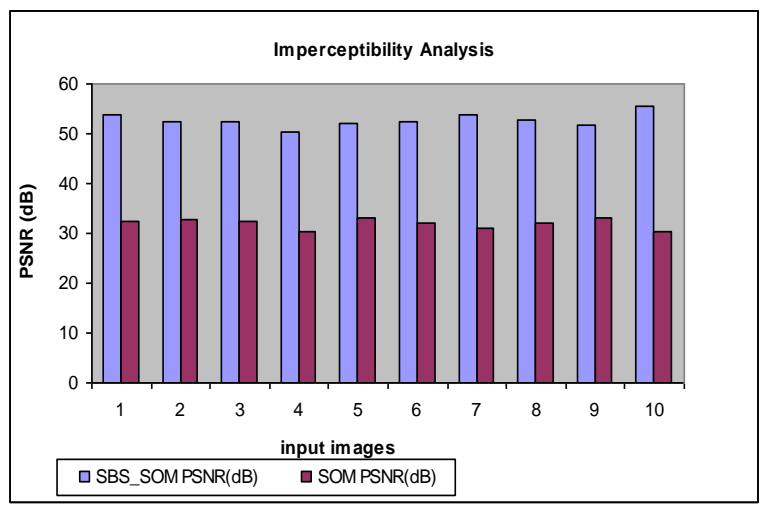

(a) 


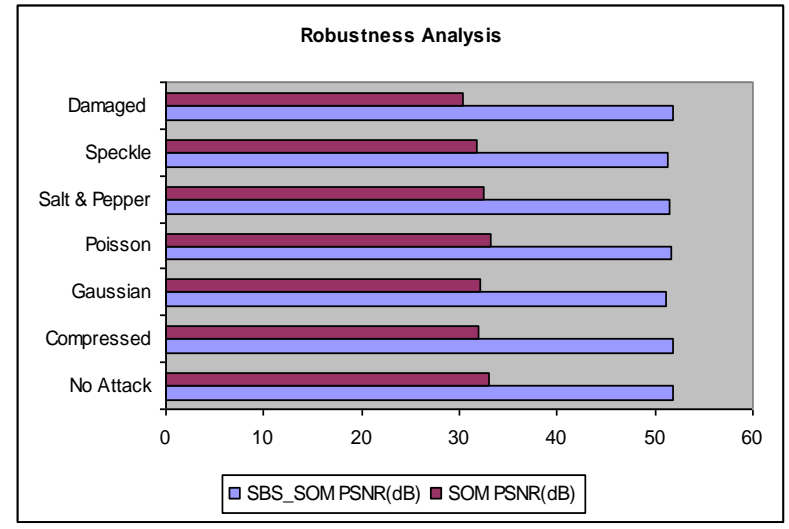

(b)

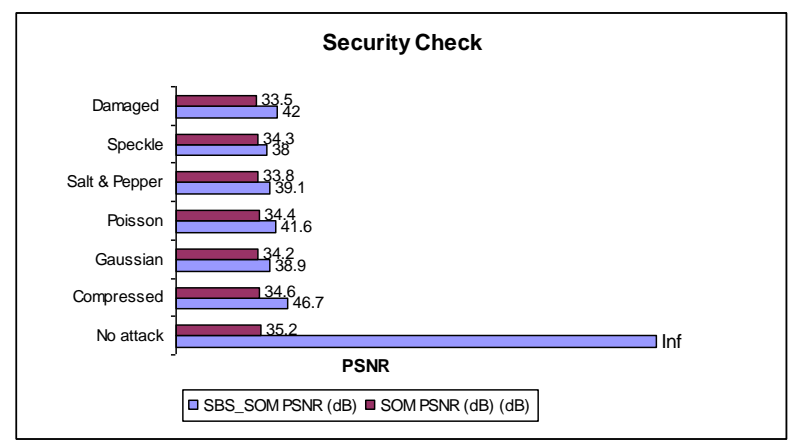

(c)

Figure 3. Fig. 4.3 (a) Imperceptibility Analysis of watermarked image (b) Robustness verification of watermark (c) Security Check of watermark removed image at the destination system

The robustness, imperceptibility and securities were analyzed and plotted as a graph to explicitly show the good quality of FFDW system with SBS_SOM. The Fig. 3 exhibits the significance of proposed watermarking. SBS_SOM based FFDW system in watermark generation is one more land mark in watermarking techniques.

\section{CONCLUSION}

SBS_SOM mined Fine Facet Digital Watermark system is an innovative technique for watermarking research. The other image, text or audio digital values are not used as digital watermark to embed. The purpose of embedding values in this process is not protecting embedding values but identifying the misuse of the host image. The watermark of each individual image is mined from the corresponding host image. Hence collecting watermark values from other image is impossible. Unauthorized users cannot detect the watermark values from watermarked image by using any statistical or correlation calculation because SBS_SOM is a complete unsupervised network trainer. FFDW is a good digital watermarking technique because it satisfies the basic requirements such as Robustness, Imperceptibility and Security. The image communication to claim authentication may use the proposed system confidently. The proposed system used DWT for the watermark embedding. In future the SBS_SOM itself will be used for embedding to prove its comprehensiveness.

\section{REFERENCES}

[1] Cheng-Ri Piao, Suenghwa Beack, Dong-Min Woo and Seung-Soo Han, "A Blind Watermarking Algorithm based on HVS and RBF Neural Network for Digital Image”, ICNC 2006, Part 1, LNCS 4221, pp. 493 496, 2006.

[2] Chenthalir Indra N, Ramaraj E, "Similar - Dissimilar Victor Measure Analysis to Improve Image Knowledge Discovery Capacity of SOM". In: Communications in Computer and Information Science, 2010, Volume 101, Part 2, 389-393, DOI: 10.1007/978-3-642-15766-0_612.

[3] Chenthalir Indra N, Ramaraj E, "Magnitude of Self Systematizing Resemblance Measures in Knowledge Mining". In: Software Technology and Engineering, 2009.pp 239-243, DOI : 10.1142 / 9789814289986_0044.

[4] Chuan-Yu Chang and Sheng-Jyun Su, "The application of a Full Counterpropagation Neural Network to Image Wateramrking", 2005.

[5] Chuan-Yu Cahng, Hung-Jen Wang, Sheng-Jyun Su, "Copyright authentication for images with a full counter propagation neural network", Expert Systems with Applications 37, 2010.

[6] Dorina Marghescu, Mikko J. Rajanen - "Assessing the Use of the SOM technique in Data Mining" - Proceedings of the 23rd International Multi-Conference on Databases and Applications, February 14-16,2005, Innsburck, Austria.

[7] Kumar, S., Raman, B., Thakur, M.: "Real Coded Genetic Algorithm based Stereo image Watermarking”. In: IJSDIA 1(1),pp.23-33 (2009).

[8] Jun Zhang, Nenchao Wang, Feng Xiong, "Hiding a Logo Watermark into the Multiwavelet Domain using Neural Networks", In the Proceedings of the 14th IEEE International Conference on Tools with Artificial Intelligence, 2002.

[9] Sujatha S S and Mohamed Sathik M "Feature Based Watermarking Algorithm by Adopting Arnold Transform". In: ICT 2010, CCIS 101, pp. 78-82, 2010. @ Springer-Verlag Berlin Heidelberg 2010.

[10] Sujatha S S and Mohamed M Sathik, “ A Novel Feature Based Blind Watermarking Scheme”. In: CiiT Inernational Journal of Digital Image Processing,Vol 2, No 7,pp.185-188, July 2010. 